\section{Patient Assessments of Skin, Joint, and Nail Disease Activity in Psoriatic Arthritis}

\section{To the Editor:}

We read with great interest the report by Cauli, et al on patient assessments of disease activity in psoriatic arthritis (PsA) ${ }^{1}$. As part of an exercise validating an instrument to assess the activity of nail psoriasis ${ }^{2}$, the modified Nail Psoriasis Severity Index (mNAPSI), we collected detailed clinical information from a cohort of 20 patients with PsA. In addition to standard measures of peripheral arthritis, functional status, quality of life, and skin psoriasis activity, this included visual analog scales (VAS) with which patients separately assessed the global activity of their PsA, as well as the activity of skin involvement, joint involvement, and nail involvement. Pairwise correlations between various outcome measures were performed using Spearman's rho. The patients had a mean age of 52.6 years, mean duration of psoriasis 18.8 years and PsA 11.0 years, mean tender joint count of 7.4/68 and swollen joint count 3.0/66, and a mean Psoriasis Area and Severity Index (PASI) score of 5.0. Correlations among selected measures are shown in Table 1.

As might be expected, there was good correlation between joint counts and the physicians' assessment of overall PsA disease activity, as well as between physician and patient global assessments. Function assessed by Health Assessment Questionnaire (HAQ) correlated with the joint counts and with the patient arthritis-specific VAS, as well as with the physical component summary (PCS) score of the Medical Outcomes Study Short Form-36 (SF-36). The SF-36 PCS also correlated with the patient arthritis-specific VAS. As has been reported, measures of joint activity and skin activity did not correlate very strongly ${ }^{3,4}$. Also, there was less strong correlation between skin activity and the PCS of the SF-36. Interestingly, different measures of skin activity (e.g., the PASI as compared to patient and physician VAS) did not correlate as strongly as might have been expected.

Table 1. Correlations among selected measures. No adjustment has been made for multiple comparisons.

\begin{tabular}{lcc}
\hline Measures Correlated & $\begin{array}{c}\text { Spearman's } \\
\text { rho }\end{array}$ & $\mathrm{p}$ \\
\hline Patient and MD global PsA activity & 0.76 & $<0.01$ \\
SJC and MD global PsA activity & 0.74 & $<0.01$ \\
TJC and MD global PsA activity & 0.68 & $<0.01$ \\
Patient global arthritis and HAQ & 0.61 & $<0.01$ \\
TJC and HAQ & 0.89 & $<0.01$ \\
SJC and HAQ & 0.54 & 0.01 \\
Patient global arthritis and SF-36 PCS & 0.75 & $<0.01$ \\
HAQ and SF-36 PCS & 0.84 & $<0.01$ \\
HAQ and PASI & 0.41 & 0.08 \\
Patient global arthritis and PASI & 0.52 & 0.02 \\
Patient global skin and PASI & 0.53 & 0.02 \\
MD global skin and PASI & 0.52 & 0.02 \\
Patient global skin and SF-36 PCS & 0.38 & 0.13 \\
PASI and SF-36 PCS & 0.56 & 0.02 \\
mNAPSI and MD nail global & 0.93 & $<0.01$ \\
mNAPSI and patient nail global & 0.55 & 0.01 \\
mNAPSI and PASI & 0.38 & 0.10 \\
mNAPSI and SF-36 PCS & 0.53 & 0.02 \\
Patient global nail and SF-36 PCS & 0.56 & 0.02 \\
mNAPSI and patient global arthritis & 0.42 & 0.07 \\
mNAPSI and TJC & 0.53 & 0.02 \\
mNAPSI and SJC & 0.44 & 0.05 \\
\end{tabular}

mNAPSI: modified Nail Psoriasis Severity Index; HAQ: Health Assessment Questionnaire; SJC: swollen joint count; TJC: tender joint count; PASI: Psoriasis Area and Severity Index; SF-36: Medical Outcome Study Short-Form 36; PCS: physical component summary.
Finally, whereas different measures of activity of nail disease (by mNAPSI and by patient global nail assessment) correlated well with each other, they did not correlate with measures of overall skin activity (PASI, patient global skin assessment). Nail involvement correlated with the PCS of the SF-36 comparable to skin involvement.

Our data support the findings by Cauli and colleagues. Accurate assessment of the various domains of PsA should include separate accounting for global severity of skin, joints, and also nails. Nail disease is increasingly recognized as of importance to patients with psoriasis; the choice of therapeutic regimen for patients with PsA or psoriasis should consider data specific to nail involvement ${ }^{3,4}$. A study by Williamson and colleagues assessed 69 patients with PsA and found correlations between nail disease severity and skin disease, enthesitis, polyarticular disease, HAQ score, higher depression and anxiety scores, and unremitting and progressive arthritis ${ }^{4}$. Scarpa and colleagues found a relationship between distal interphalangeal (DIP) arthritis in patients with PsA and the duration of nail psoriasis, although there was not a statistically significant correlation between the distribution of DIP arthritis and onychopathy ${ }^{5}$.

In our cohort, DIP joint arthritis was not associated with nail involvement, but active DIP arthritis was not common. In addition, we found no strong correlation between mNAPSI and overall measures of arthritis, including patient global VAS for arthritis activity, tender joint count, and swollen joint count.

We therefore agree with Cauli and colleagues that assessment of the various domains of PsA should include separate VAS measures for the global severity of skin, joints, and also nail involvement.

ARTHUR KAVANAUGH, MD, Division of Rheumatology, University of California San Diego, San Diego, CA, USA; TERESA CATALAN, MD, Inmunoreumatologia, Instituto Mexicano del Seguro Social, Mexico City, Mexico; SARAH CASSELL, MD, Division of Rheumatology, University of California San Diego, 9500 Gilman Drive, La Jolla, CA, USA 92037-0943.E-mail: akavanaugh@ucsd.edu

\section{REFERENCES}

1. Cauli A, Gladman DD, Mathieu A, Olivieri I, Porru G, Tak PP, et al. Patient global assessment in psoriatic arthritis: A multicenter GRAPPA and OMERACT study. J Rheumatol 2011;38:898-903.

2. Cassell S, Bieber J, Rich P, Tutuncu Z, Lee SJ, Kalunian K, et al The modified Nail Psoriasis Severity Index: Validation of an instrument to assess psoriatic nail involvement in patients with psoriatic arthritis. J Rheumatol 2007;34:123-9.

3. Cassell SE, Kavanaugh A. Therapies for psoriatic nail disease. A systematic review. J Rheumatol 2006;33:1452-6.

4. Williamson L, Dalbeth N, Dockerty JL, Gee BC, Weatherall R, Wordsworth BP. Extended report: Nail disease in psoriatic arthritis - clinically important, potentially treatable and often overlooked. Rheumatology 2004;43:790-4.

5. Scarpa R, Manguso F, Oriente A, Peluso R, Attno M, Oriente P. Is the involvement of distal interphalangeal joint in psoriatic patients related to nail psoriasis? Clin Rheumatol 2004;23:27-30.

J Rheumatol 2012;39:3; doi:10.3899/jrheum.110839

Personal non-commercial use only. The Journal of Rheumatology Copyright ()$^{2012 . ~ A l l ~ r i g h t s ~ r e s e r v e d . ~}$ 